\title{
POSE Algorithms for Automated Docking
}

\author{
Andrew F. Heaton and Richard T. Howard* \\ NASA Marshall Space Flight Center, Huntsville, AL 35812
}

\begin{abstract}
POSE (relative position and attitude) can be computed in many different ways. Given a sensor that measures bearing to a finite number of spots corresponding to known features (such as a target) of a spacecraft, a number of different algorithms can be used to compute the POSE. NASA has sponsored the development of a flash LIDAR proximity sensor called the Vision Navigation Sensor (VNS) for use by the Orion capsule in future docking missions. This sensor generates data that can be used by a variety of algorithms to compute POSE solutions inside of 15 meters, including at the critical docking range of approximately 1-2 meters. Previously NASA participated in a DARPA program called Orbital Express that achieved the first automated docking for the American space program. During this mission a large set of high quality mated sensor data was obtained at what is essentially the docking distance. This data set is perhaps the most accurate truth data in existence for docking proximity sensors in orbit. In this paper, the flight data from Orbital Express is used to test POSE algorithms at 1.22 meters range. Two different POSE algorithms are tested for two different Fields-of-View (FOVs) and two different pixel noise levels. The results of the analysis are used to predict future performance of the POSE algorithms with VNS data.
\end{abstract}

Keywords: VNS, POSE, AR\&D, automated docking, sensor, algorithm, flash LIDAR

\section{INTRODUCTION}

Spacecraft maneuvers depend on accurate information. The accuracy of the information is even more important when the spacecraft is maneuvering relative to another object in space. There are different types of sensors used to help measure the relative position and attitude, or POSE, between one spacecraft and another, but all of the sensors require algorithms to allow them to properly compute the POSE from the basic measurements they take. In general, the sensor measurements consist of a set of points on the target vehicle, such as the centroids of the spots seen on a target or particular features on the target vehicle. The POSE algorithm takes those sensor measurements as inputs and computes the relative position and attitude between the sensor and the target.

The VNS was designed to provide data that would be sufficient to compute relative 6-Degree-of-Freedom (DOF) (3DOF relative position and 3-DOF relative attitude) data when used with an appropriate target. The VNS and DragonEye space qualified flash LIDAR systems both provide intensity and range images of targets in their FOVs, but that image data must then be processed to be of use to a guidance, navigation, and control (GN\&C) system.

\section{ANALYSIS}

\subsection{POSE Algorithms}

Various POSE algorithms have been considered by the VNS team. These algorithms have been narrowed down to three, which will be described in more detail below, and two of those will be used with previously collected flight sensor data. Each of these algorithms can combine Time-Of-Flight (TOF) LIDAR range estimates for the spot locations with the POSE solutions. These alternate methods of solution that incorporate LIDAR range are not considered in this analysis, since no LIDAR range is available from Orbital Express data. Furthermore, the current VNS LIDAR range error is perhaps too high to be of use at the docking position, and it may not be useable. At the time of publication of this paper, it is very possible that for docking range estimates a solution based solely on POSE is likely. Regardless of the eventual nature of the solution method employed by VNS at the docking range, this paper focuses solely on the

*ricky.howard@nasa.gov; phone 256-544-3536; fax 256-544-6894 
POSE solution. In the Orion spacecraft, the VNS data will be processed by the Video Processing Unit (VPU). That unit consists of a set of processors, Field Programmable Gate Arrays (FPGAs), and software that can take sensor and video data and process it in real time. There are two POSE algorithms that will be used by the VPU that are discussed in detail in this paper. The first is called P3P. P3P is a method of finding a solution from the location of three and only three known locations on the target. It is based on the method of Finsterwalder [1] as adapted by Haralick et al [2]. The problem is set up as a geometry problem and solved with a cubic and two quadratic polynomials.

The other primary method of solving the POSE problem for VNS is called the Least Squares POSE or LSP. LSP consists of a way of combining more than three points together when they are available. It also follows the method outlined in Haralick. If LIDAR data is available for more than three spots, a third algorithm, Weighted Least Squares Program or WLSP is used. As noted above, this algorithm is beyond the scope of this paper. Due to the fixed error from LIDAR TOF solutions, which become a bigger percentage of the solution at close ranges, WLSP is thought to be more effective at improving the POSE solution at longer ranges. Since this paper focuses on the minimum range, the LIDAR range solutions are omitted and WLSP is not simulated.

The P3P algorithm follows the solution method of Haralick [2]. In this method, the camera measures exactly three image points on the target vehicle. These image points can be combined with information about the known geometry between those points on the target vehicle and the Law of Cosines can be used to form the following equations,

$$
\begin{aligned}
& \mathrm{R}_{1}{ }^{2}+\mathrm{R}_{2}{ }^{2}-2 \mathrm{R}_{1} \mathrm{R}_{2} \cos \theta_{12}-\mathrm{r}_{12 \mathrm{~T}}{ }^{2}=0 \\
& \mathrm{R}_{1}{ }^{2}+\mathrm{R}_{3}{ }^{2}-2 \mathrm{R}_{1} \mathrm{R}_{3} \cos \theta_{13}-\mathrm{r}_{13 \mathrm{~T}}{ }^{2}=0 \\
& \mathrm{R}_{2}{ }^{2}+\mathrm{R}_{3}{ }^{2}-2 \mathrm{R}_{2} \mathrm{R}_{3} \cos \theta_{23}-\mathrm{r}_{23} \mathrm{~T}^{2}=0
\end{aligned}
$$

where $\theta_{\mathrm{ij}}$ represents the relative angles between the points in the image, $\mathrm{r}_{\mathrm{ij}}$ represents the physical distances between the points on the target vehicle, and $\mathrm{R}_{\mathrm{i}}$ is the range from the sensor optical center to the physical points on the target vehicle (which is the translational solution desired). The P3P method uses a polynomial to solve these equations, which consist of three equations with three unknowns $\left(\mathrm{R}_{1}, \mathrm{R}_{2}\right.$, and $\left.\mathrm{R}_{3}\right)$. Other methods of solving the equations have been tried with other sensors [3].

\subsection{Orbital Express Flight Data Overview}

In this section, flight results from the Orbital Express mission are used as a basis of simulation and comparison with VNS POSE Estimation algorithms. Orbital Express was launched in March of 2007 and operated until August of 2007. During operations, fifteen different data sets of proximity sensor data were collected while the chase and target vehicles were mated. These data sets represent a source of highly accurate truth data due to the tight tolerance on the Orbital Express docking mechanism. The variation in bias from set to set is small and significantly less than the Orbital Express docking sensor specification. The Orbital Express primary docking sensor was the Advanced Video Guidance Sensor or AVGS.

It is important to stress that in the experience of the authors, the test data for the AVGS while docked on Orbital Express can be considered the most accurate truth data in existence for this type of sensor. Ground testing accuracy has limitations [4], which can be reduced to the following statement: proximity sensors used for docking generally are required to be so accurate that any source of truth data for ground testing is likely to be of the same order or less in accuracy. This is particularly true considering that all known proximity sensors use lenses and thus optics plays a role. Attempting to measure the focal plane of a sensor in situ, for example, has proven difficult in the past. There are also other sources of error and problems with getting accurate truth data in ground testing.

By contrast, the docking mechanism on Orbital Express had a tight tolerance that was automatically repeated every time an operation was performed, and in the process hours of data were collected in the mated position. In fact, three of the fifteen tests were specifically conducted to calibrate or characterize the performance of the Orbital Express chase vehicle proximity sensor suite. Two sets of ground data in the mated position were also taken in the clean room in Titusville, Florida, prior to launch, further increasing the value of the on-orbit data set by providing a pre-launch set of data for 
comparison. Finally, the value of the on-orbit data is significantly improved over any ground set of data by virtue of the fact that it was taken in the relevant environment and incorporated environmental effects such as vacuum, on-orbit thermal effects and on-orbit lighting (sunlight, shadow, darkness, and earth background).

The key to using AVGS Orbital Express data to test VNS algorithms is to convert the AVGS inputs into the VNS input format used by the simulation software. Both sensors use target spot locations measured by the camera as inputs into the POSE Estimation algorithms. These inputs were preserved along with the AVGS output data and so are available for use with the VNS POSE Estimation algorithms. However, the basic VNS POSE Estimation software is not currently configured to receive large inputs of target spot locations in batch mode. Instead, the VNS simulation software Monte Carlo POSE Estimation (MCPE) can accept as user input two truth solutions and a pixel noise parameter. MCPE then randomly selects a truth case that is between the two solutions, calculates target spot locations for the selected truth case, adds pixel noise to the simulated target spots, and finally regenerates the solution with pixel noise. The user can choose an arbitrary number of these generated solutions. For the purposes of testing in this paper we use 1000 cases.

\subsection{Flight Data Conversion to VNS Format}

During the Orbital Express mission, several different scenarios were run. The scenarios involving free-flight were Scenario 2-1, Scenario 3-1, Scenario 5-1, Scenario 7-1, Scenario 8-2, and EOL (End-of-Life) [5]. In addition, there were some other sensor checkouts that did not involve undocking and redocking, so the AVGS and the target were in the same position relative to one another as they had been the last time the sensor took measurements. The sensor checkouts were part of different utility runs with such names as AT-UTIL-25. The sensor data from the AVGS compared very nicely with the Kalman filter that was used to blend all of the spacecraft sensor data [6].

It was somewhat of a challenge to figure out how to make the Orbital Express data from the AVGS fit into MCPE without major code modifications. In order to discuss how this was accomplished, it is necessary to describe the Orbital Express AVGS data that was available. Table 1 and Table 2 present the results of all fifteen on-orbit data samples for the AVGS. These samples represent the truth data with one-sigma variations for each set. Notice also that the "truth position" wanders slightly from one position to the next. The slight variation between samples can be considered a bias, and represents the variation in the docking mechanism from case to case (there may be other factors causing the slight variations; but for the purposes of this paper, given how small the variations are, we assume that the differences are all due to mechanical variations in the docking mechanism due to tolerance, thermal variations, and mechanical deformation). The slight variation from case to case provides the bounds of the two truth solutions required by MCPE. The total minimum and maximum values from all fifteen cases for each solution parameter were selected and the results are tabulated in Table 3.

Table 1: Orbital Express AVGS Mated Solution Data (Median Values)

\begin{tabular}{|l|c|c|c|c|c|c|c|c|c|}
\hline & $\begin{array}{l}\text { End of } \\
\text { Life pre }\end{array}$ & $\begin{array}{l}\text { Scn 8-2 } \\
\text { pre }\end{array}$ & $\begin{array}{l}\text { Scn 8-2 } \\
\text { post }\end{array}$ & $\begin{array}{l}\text { Scn 7-1 } \\
\text { pre }\end{array}$ & $\begin{array}{l}\text { Scn 7- } \\
\text { 1 post }\end{array}$ & $\begin{array}{l}\text { Scn 5-1 } \\
\text { pre }\end{array}$ & $\begin{array}{l}\text { Scn 5-1 } \\
\text { post }\end{array}$ & $\begin{array}{l}\text { Scn 3- } \\
\text { 1 pre }\end{array}$ & $\begin{array}{l}\text { Scn 3-1 } \\
\text { post }\end{array}$ \\
\hline Duration [min] & 3.04 & 3.01 & 16.29 & 3.03 & 13.35 & 3.03 & 1.05 & 3.03 & 0.99 \\
\hline & & & & & & & & & \\
\hline Range[m] & 1.220 & 1.220 & 1.220 & 1.220 & 1.220 & 1.219 & 1.220 & 1.220 & 1.220 \\
\hline Azimuth [deg] & -0.008 & -0.009 & -0.004 & -0.009 & -0.010 & -0.011 & -0.015 & -0.006 & 0.003 \\
\hline Elevation [deg] & -0.021 & -0.024 & -0.034 & -0.025 & -0.032 & -0.023 & -0.022 & -0.028 & -0.033 \\
\hline Pitch [deg] & 0.000 & 0.000 & 0.091 & 0.044 & 0.024 & 0.021 & 0.052 & 0.004 & 0.021 \\
\hline Yaw [deg] & -0.028 & -0.049 & -0.014 & -0.105 & 0.000 & 0.000 & 0.000 & -0.052 & -0.024 \\
\hline Roll [deg] & 0.059 & 0.066 & 0.035 & 0.098 & 0.084 & 0.049 & 0.063 & 0.091 & 0.105 \\
\hline
\end{tabular}


Table 2: Additional Orbital Express AVGS Mated Solution Data

\begin{tabular}{|l|c|c|c|c|c|c|}
\hline & $\begin{array}{l}\text { Scn 2-1 } \\
\text { pre }\end{array}$ & $\begin{array}{l}\text { Scn 2-1 } \\
\text { post }\end{array}$ & $\begin{array}{l}\text { Scn 1-1 } \\
\text { post }\end{array}$ & $\begin{array}{l}\text { AT_UTIL- } \\
\mathbf{2 5}(\mathbf{1 s t})\end{array}$ & $\begin{array}{l}\text { AT_UTIL- } \\
\text { 25(2nd) }\end{array}$ & $\begin{array}{l}\text { Sensor } \\
\text { Stress Test } \\
\text { (AC3) }\end{array}$ \\
\hline Duration [min] & 3.02 & 1.04 & 0.46 & 43.98 & 22.10 & 309.56 \\
\hline & & & & & & \\
\hline Range [m] & 1.220 & 1.220 & 1.220 & 1.220 & 1.220 & 1.220 \\
\hline Azimuth [deg] & -0.009 & -0.006 & -0.010 & -0.009 & -0.007 & -0.010 \\
\hline Elevation [deg] & -0.026 & -0.025 & -0.032 & -0.024 & -0.023 & -0.023 \\
\hline Pitch [deg] & 0.000 & 0.049 & 0.053 & 0.028 & 0.035 & 0.010 \\
\hline Yaw [deg] & -0.045 & -0.056 & -0.045 & -0.038 & 0.000 & 0.000 \\
\hline Roll [deg] & 0.098 & 0.126 & 0.122 & 0.094 & 0.101 & 0.084 \\
\hline
\end{tabular}

A further complication is that the solution of the VPU is in a different format from that of the AVGS. The attitude portion of the solution is identical, but the translational range of the VNS is output as Cartesian coordinates (X, Y, and Z) while the AVGS outputs the same information in a range/bearing angle format (Range, Azimuth, and Elevation). Thus, for purposes of comparing the respective solutions, the AVGS Range, Azimuth, and Elevation are converted to VNS X, Y, and Z. The conversion is straightforward, and the results appear in Table 3. Table 3 presents the upper and lower bounds of the AVGS case-to-case bias for all fifteen samples in the VNS coordinate frame, with the Euler angle data repeated for convenience. Thus, when MCPE generates a random sample between the two sets of solution data (i.e. the Minimum data set and the Maximum data set) in Table 3, it is essentially selecting a random bias for the given run. Each of the $1000 \mathrm{MCPE}$ solutions will then have a different fixed random bias.

Table 3: Minimum and Maximum AVGS Solutions in Orbital Express Mated Positions

\begin{tabular}{|c|c|c|}
\hline Parameter & Min & Max \\
\hline $\mathbf{X}(\mathbf{m m})$ & -0.319 & 0.0639 \\
\hline Y (mm) & -0.723 & -0.447 \\
\hline Z (mm) & 1219 & 1220 \\
\hline Pitch (deg) & -0.016 & 0.086 \\
\hline Yaw (deg) & -0.050 & 0.016 \\
\hline Roll (deg) & 0.035 & 0.126 \\
\hline
\end{tabular}

The next step is to convert the noise of the AVGS target spots into VNS target spots in the appropriate format. AVGS input target spot locations are available with the solution data as ordered pairs of spot locations in an Azimuth-Elevation format. There are four spot locations total. The VNS simulator's noise parameter input is Root Sum Square (RSS) pixel noise. The AVGS angular spot location coordinates are converted to VNS x-pixel and y-pixel coordinates with a simple linear gain, and the two VNS pixel coordinates combined into the RSS pixel noise used by MCPE. Table 4 lists the AVGS spot centroid noise levels and the corresponding Elevation and Azimuth noise in degrees. MCPE takes this as input a one-sigma pixel noise and applies it to simulated spot locations using a random white-noise Gaussian distribution. This noise must be calculated on the basis of the size of the FOV and number of pixels for a given sensor. In this analysis both the AVGS FOV and VNS FOV are simulated, so we describe the relevant pixel noise used for each below. The noise is converted from Table 4 into a single RSS pixel noise parameter for input into MCPE. 
Table 4: AVGS RSS Spot Noise from Orbital Express Mated Positions

\begin{tabular}{|c|c|c|c|}
\hline & Azimuth & Elevation & Units \\
\hline Spot 1 & 0.004218 & 0.003557 & $\mathrm{deg}$ \\
\hline Spot 2 & 0.004359 & 0.003885 & $\mathrm{deg}$ \\
\hline Spot 3 & 0.004567 & 0.003640 & $\mathrm{deg}$ \\
\hline Spot 4 & 0.003811 & 0.003649 & $\mathrm{deg}$ \\
\hline RSS & 0.004239 & 0.003683 & $\mathrm{deg}$ \\
\hline
\end{tabular}

As mentioned above, both the AVGS and VPU convert target spot locations into solutions. The reference points used by the AVGS are described as the "target matrix" by AVGS programmers. The target matrix is simply a list of the locations of the AVGS spots in the body frame of the target assembly used by AVGS. This target assembly consists of corner cube reflectors and appears in Fig. 1. These locations have been converted into the VNS target body coordinate frame used by MCPE and appear in Table 5. The spot locations are loaded in the order they were entered into MCPE, and not the OE AVGS nomenclature. Fig. 1 also shows the geometric relationship of the spots in the VNS coordinate system as numbered in Table 4.

Table 5: AVGS Target Spot Locations in VNS Coordinates (cm)

\begin{tabular}{|c|c|c|c|}
\hline Spot \# & $\mathbf{X}$ & $\mathbf{Y}$ & $\mathbf{Z}$ \\
\hline $\mathbf{1}$ & 6.32765 & -0.03168 & -6.26298 \\
\hline $\mathbf{2}$ & -6.36587 & -0.06732 & -6.19930 \\
\hline $\mathbf{3}$ & -0.03398 & 6.29857 & -6.29040 \\
\hline $\mathbf{4}$ & 0.00000 & 0.00000 & -1.17856 \\
\hline
\end{tabular}




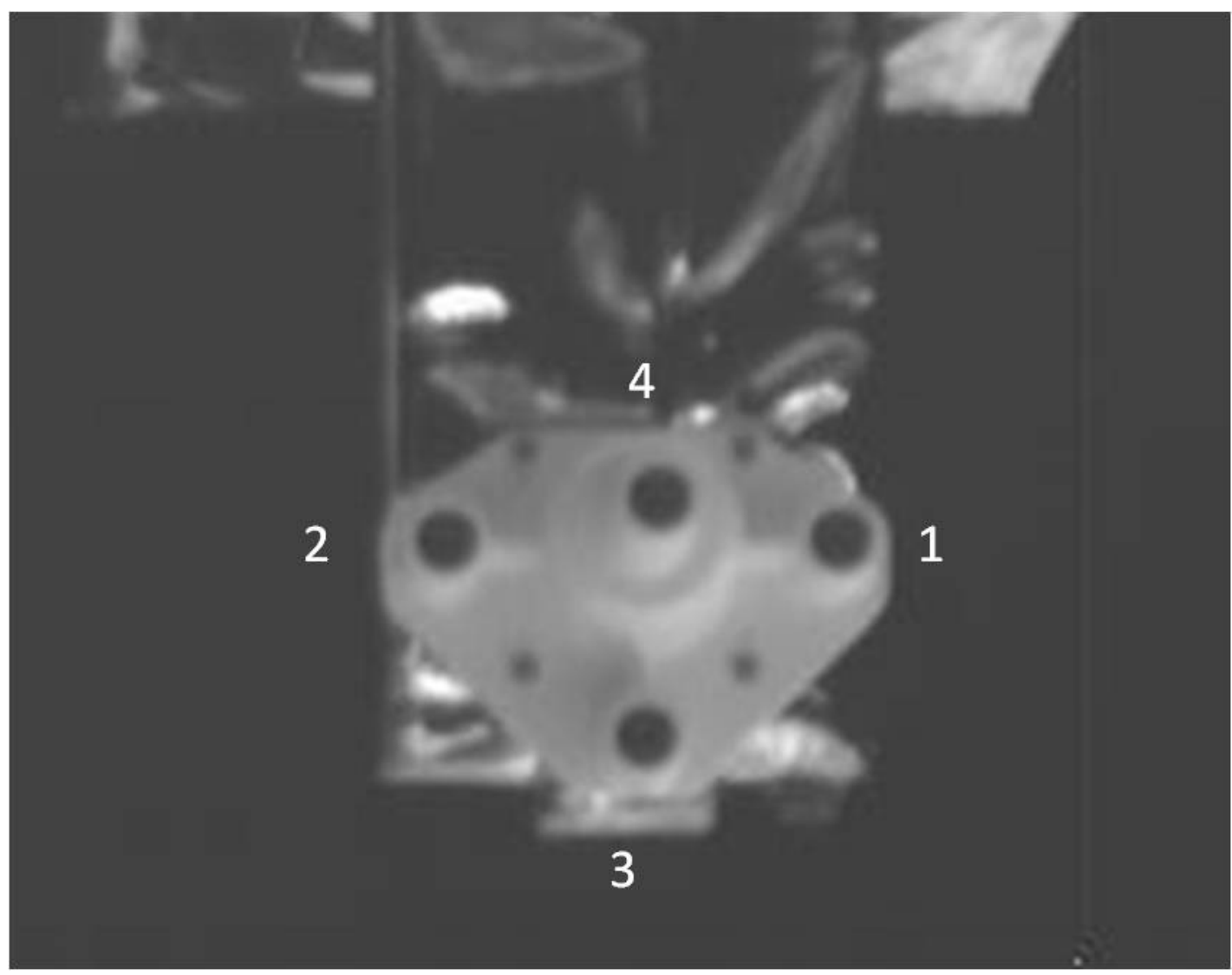

Fig. 1: AVGS Orbital Express Target Assembly with VNS Target Spot Numbering Scheme

\subsection{Test Case Description}

The purpose of this effort is to use Orbital Express data to characterize the performance of the POSE algorithms expected to be used by the VPU with VNS data. One way to do this is to simulate the AVGS FOV and imager pixel size in MCPE and compare the results to those obtained on orbit. The only difference in the two cases would be the POSE algorithms. As detailed above, AVGS uses the Inverse Perspective while the VPU uses P3P. Each of these algorithms uses only three points. This will be Test Case \#1.

The VPU can also use the LSP algorithm to incorporate more than three spots. Since there were four spots available on Orbital Express, it makes sense to see if LSP can improve the solution by using the fourth spot. This will be Test Case \#2.

Next, it's interesting to see how the VNS FOV and imager pixel size would have worked for Orbital Express, and to see if it would meet specification. Thus, Test Cases \#3-4 repeat Test Cases \#1-2 with the VNS FOV and imager pixel size replacing that of the AVGS.

The spot noise generated by the AVGS on-orbit is captured in Table 5. This noise is in degrees and on the Azimuth and Elevation axes. Table 6 converts this noise into the RSS combined axis pixel noise for all of the Test Cases. 
Table 6: Test Case Description

\begin{tabular}{|c|c|c|c|c|}
\hline Test Case & Pixel Noise & FOV (deg) & \# of Pixels & Algorithm \\
\hline $\mathbf{1}$ & 0.07168 & 20 & 256 & P3P (3 Spots) \\
\hline $\mathbf{2}$ & 0.07168 & 20 & 256 & LSP (4 Spots) \\
\hline $\mathbf{3}$ & 0.10000 & 20 & 256 & P3P (3 Spots) \\
\hline $\mathbf{4}$ & 0.10000 & 20 & 256 & LSP (4 Spots) \\
\hline
\end{tabular}

\section{DISCUSSION OF RESULTS}

\subsection{Overview}

This section will present the results achieved for the test cases outlined above. The test cases are organized in three groups of two, with the distinguishing feature between the two in each group being the particular POSE algorithm simulated. All the test cases will therefore be discussed in groups of two.

\section{Table 7: AVGS Flight Data Standard Deviations}

\begin{tabular}{|l|l|}
\hline$X(\mathrm{~mm})$ & 0.078 \\
\hline$Y(\mathrm{~mm})$ & 0.079 \\
\hline$Z$ (mm) & 0.564 \\
\hline Pitch (deg) & 0.035 \\
\hline Yaw (deg) & 0.037 \\
\hline Roll (deg) & 0.027 \\
\hline
\end{tabular}

Before presenting the results of the simulations, we first present in Table 7 the AVGS truth data from the mated Orbital Express positions. Table 7 presents the one-sigma variations of the AVGS solution from the on-orbit flight data presented in Tables 1 and 2. The AVGS Range-Az-El translational portion of the solution has been converted into the VNS X-Y-Z solution to allow a one-to-one comparison. All four test cases will be compared to this result, and to each other.

\subsection{VNS Image Spot Test Cases with AVGS Pixel Noise}

Tables 8 and 9 display the results from the VNS FOV and imager number of pixels. There is a roughly 10-12\% improvement from the 3-spot (P3P) solution to the 4-spot (LSP) solution. The data is roughly five times less accurate than the AVGS truth data in Table 7. The combination of $75 \%$ fewer pixels in the VNS and the $25 \%$ larger FOV of the VNS compared to the AVGS most likely accounts for this difference in accuracy. 
Table 8: Test Case 1 Results

\begin{tabular}{|c|c|}
\hline Parameter & 1-Sigma Error \\
\hline $\mathbf{X ~ ( m m )}$ & 0.2000 \\
\hline Y (mm) & 0.2000 \\
\hline $\mathbf{Z}$ (mm) & 1.7000 \\
\hline Pitch (deg) & 0.1729 \\
\hline Yaw (deg) & 0.1609 \\
\hline Roll (deg) & 0.0766 \\
\hline
\end{tabular}

Table 9: Test Case 2 Results

\begin{tabular}{|c|c|}
\hline Test Case & 1-Sigma Error \\
\hline $\mathbf{X}(\mathbf{m m})$ & 0.1000 \\
\hline Y (mm) & 0.1000 \\
\hline $\mathbf{Z}$ (mm) & 1.5000 \\
\hline Pitch (deg) & 0.1528 \\
\hline Yaw (deg) & 0.1477 \\
\hline Roll (deg) & 0.0698 \\
\hline
\end{tabular}

\subsection{VNS Image Spot Test Cases with Specified Pixel Noise}

The next two test cases repeat the previous two with the VNS specified pixel noise of 0.1 pixels substituted for the flight-data-derived value of 0.07168 used above. The results of the MCPE Monte Carlo simulations using P3P (Test Case 10) and LSP (Test Case 11) appear in Tables 10 and 11. It can be seen that the result of using a higher pixel noise is a corresponding increase in inaccuracy, at a level commensurate with the increase in noise.

Table 10: Test Case 3 Results

\begin{tabular}{|c|c|}
\hline Parameter & 1-Sigma Error \\
\hline X (mm) & 0.2000 \\
\hline Y (mm) & 0.2000 \\
\hline Z (mm) & 2.1000 \\
\hline Pitch (deg) & 0.2312 \\
\hline Yaw (deg) & 0.2140 \\
\hline Roll (deg) & 0.0966 \\
\hline
\end{tabular}

Table 11: Test Case 4 Results

\begin{tabular}{|c|c|}
\hline Parameter & 1-Sigma Error \\
\hline $\mathbf{X}(\mathbf{m m})$ & 0.2000 \\
\hline $\mathbf{Y ~ ( m m )}$ & 0.2000 \\
\hline $\mathbf{Z}$ (mm) & 2.2000 \\
\hline Pitch (deg) & 0.2173 \\
\hline Yaw (deg) & 0.2050 \\
\hline Roll (deg) & 0.0949 \\
\hline
\end{tabular}


Although at the time of publication of this paper no official specification of the VPU or VNS has been published, some notional values from an unofficial source appear in Table 12. These values are included only for comparison, and should not be considered official in any way.

Table 12: Notional Specification for POSE solutions from VNS data

\begin{tabular}{|c|c|c|c|c|}
\hline $\begin{array}{l}\text { Distance } \\
\text { To Target }\end{array}$ & $\begin{array}{c}\text { Lateral } \\
\text { Position } \\
\text { Error }\end{array}$ & $\begin{array}{c}\text { Range } \\
\text { Error }\end{array}$ & $\begin{array}{c}\text { Relative } \\
\text { Attitude } \\
\text { Error } \\
\text { (pitch and } \\
\text { yaw axis) }\end{array}$ & $\begin{array}{c}\text { Relative } \\
\text { Attitude } \\
\text { Error } \\
\text { (roll axis) }\end{array}$ \\
\hline $2 \mathrm{~m}$ & $\pm 1.3 \mathrm{~mm}( \pm 0.051 \mathrm{in})$ & $\begin{array}{c} \pm 7.6 \mathrm{~mm} \\
( \pm 0.30 \mathrm{in})\end{array}$ & \pm 0.14 degree & \pm 0.34 degree \\
\hline$(6.56 \mathrm{ft})$ & $\pm 4.7 \mathrm{~cm}$ & $\begin{array}{c} \pm 4.4 \mathrm{~cm} \\
( \pm 1.73 \mathrm{in})\end{array}$ & \pm 0.63 degree & \pm 0.61 degree \\
\hline $15 \mathrm{~m}$ & $( \pm 9.2 \mathrm{ft})$ & $( \pm 1.85 \mathrm{in})$ & & \\
\hline
\end{tabular}

Viewing the values listed in Table 12, we can see that the VNS as-is would not meet specification for the pitch or yaw error at 2 meters using either the flight-data-derived pixel noise of 0.07168 or the specified noise of 0.1000 . However, all the other parameters would be well within specification. The pitch and yaw would have approximately a $20 \%$ increase in error with the flight-data-derived noise level and a roughly 50\% increase in error with the VNS-specified pixel noise.

\section{CONCLUSIONS AND FUTURE WORK}

Certain selected truth data from Orbital Express has been used to simulate results for the VNS sensor. The accuracy of the POSE solutions that can be calculated from VNS data is seen to have a strong dependency on the FOV and number of imager pixel differences from the AVGS. Two levels of pixel noise were simulated. The first is that seen by the AVGS on orbit, adjusted for the VNS FOV and number of pixels. The specified value is higher and leads to a corresponding loss of accuracy in the solution.

A comparison to a notional specification shows that the VNS would have trouble meeting this specification if the target used by the VNS were identical to that used for Orbital Express and for the proposed VNS FOV, number of pixels, and pixel noise. Since this specification is notional, nothing is implied about the ability of the VNS to meet any specification. Rather, this investigation gives an indication of what level of accuracy could be expected for the given FOV, number of pixels and specified pixel noise of the VNS. A target was installed on the International Space Station (ISS) to provide reflectors at known locations for use by the VNS [7]. This target has a baseline that is more than double that used for the AVGS and a pole that is approximately six times as tall. This target size will allow for much more accurate POSE computations since the pixel noise will be a much smaller percentage of the spot spread, and the taller pole causes more motion for a given angle, making a sensor more sensitive to measuring the relative pitch and yaw.

It should be noted in closing that pixel noise is only one potential source of error for proximity sensors used for docking, and that other sources of error can be far larger. Further investigations could consider modeling other error sources using either existing simulations like MCPE or other existing software tools available to the authors. At the time this paper is submitted to the conference, the launch date of STS-134 is April 29, 2011, and this paper is scheduled to be presented a few days earlier. STS-134 includes a Detailed Test Objective (DTO) called STORRM (Sensor Test for Orion RelNAV Risk Mitigation) which will test a version of the VNS in open loop during the shuttle's approach to the ISS. The results of this test could be used in conjunction with the results of this analysis for future studies. 


\section{ACKNOWLEDEMENTS}

Both authors appreciate the work NASA, DARPA, Boeing, Orbital Sciences Corporation, Lockheed-Martin, and Ball Aerospace have done in developing sensors and technologies for automated docking. The authors also thank Preston Faiks of Lockheed-Martin for use of and assistance with VNS POSE simulations.

\section{REFERENCES}

[1] Finsterwalder, S., and W. Scheufele, "DasRuckwartseinsclineiden im Raum" SebastianFinsterwalder ium 75. Geburtstage, Verlag Herbert Wichmann, Berlin, Germany, pp. 86-100 (1937).

[2] Haralick, R., Lee, C., et al, "Analysis and Solutions of The Three Point Perspective POSE Estimation Problem", Computer Vision and Pattern Recognition, Proceedings CVPR, '91, June 3-9 (1991).

[3] Calhoun, P. and Dabney, R., "A Solution to the Problem of Determining the Relative 6 DOF State for Spacecraft Automated Rendezvous and Docking", Proceedings of SPIE Space Guidance, Control, and Tracking II, pp. 175-184 (1995).

[4] Heaton, A. F., Howard, R. T., and Pinson, R. M., "Orbital Express AVGS Validation and Calibration for Automated Rendezvous", Proceedings of AIAA Guidance, Navigation, and Control Conference, Oahu, Hawaii, August (2008).

[5] Leinz, M. R., Chen, C., et al, "Orbital Express Automated Rendezvous and Capture Sensor System (ARCSS) Test Results", Proceedings of SPIE Sensors and Systems for Space Applications (2008).

[6] LeCroy, J., Hallmark, D., Scott, P., and Howard, R., "Comparison of Navigation Solution for Autonomous Spacecraft from Multiple Sensor Systems", Proceedings of SPIE Sensors and Systems for Space Applications (2008).

[7] http://www.nasaspaceflight.com/2010/04/sts-131-discovery-undocking-storrm-tridar-highlighted/ 